\title{
Article \\ Mandibular Reconstruction with Bridging Customized Plate after Ablative Surgery for ONJ: A Multi-Centric Case Series
}

\author{
Salvatore Battaglia ${ }^{1}$, Francesco Ricotta ${ }^{2}\left(\mathbb{D}\right.$, , Salvatore Crimi ${ }^{1, *} *$, Rosalia Mineo ${ }^{3}$, Fabio Michelon ${ }^{4}$, \\ Achille Tarsitano $^{2} \mathbb{D}$, Claudio Marchetti ${ }^{2} \mathbb{D}$ and Alberto Bianchi ${ }^{1}[\mathbb{C}$ \\ 1 Maxillofacial Surgery Unit, Department of CHIRMED, Policlinico San Marco University Hospital, \\ University of Catania, 95124 Catania, Italy; salvbatt89@yahoo.it (S.B.); alberto.bianchi@unict.it (A.B.) \\ 2 Maxillofacial Surgery Unit, Department of Biomedical and Neuromotor Sciences, University of Bologna, \\ 40126 Bologna, Italy; fraricot@gmail.com (F.R.); achille.tarsitano2@unibo.it (A.T.); \\ claudio.marchetti@unibo.it (C.M.) \\ 3 Mt Ortho srl, Via Fossa Lupo sn Aci Sant'Antonio, 95025 Catania, Italy; rosalia.mineo@mtortho.com \\ 4 Sintac S.r.l., Biomedical Engineering, Via Ragazzi del '99, 13, 38123 Trento, Italy; fabio.michelon@sintac.it \\ * Correspondence: torecrimi@gmail.com
}

check for updates

Citation: Battaglia, S.; Ricotta, F.; Crimi, S.; Mineo, R.; Michelon, F.; Tarsitano, A.; Marchetti, C.; Bianchi, A. Mandibular Reconstruction with Bridging Customized Plate after Ablative Surgery for ONJ: A Multi-Centric Case Series. Appl. Sci. 2021, 11, 11069. https://doi.org/ 10.3390/app112211069

Academic Editor: Bruno Chrcanovic

Received: 1 October 2021

Accepted: 16 November 2021

Published: 22 November 2021

Publisher's Note: MDPI stays neutral with regard to jurisdictional claims in published maps and institutional affiliations.

Copyright: (c) 2021 by the authors. Licensee MDPI, Basel, Switzerland. This article is an open access article distributed under the terms and conditions of the Creative Commons Attribution (CC BY) license (https:// creativecommons.org/licenses/by/ $4.0 /)$.
Featured Application: Accuracy evaluation of CAD/CAM bridging plate for mandibular reconstruction in patients not feasible for bony reconstruction.

\begin{abstract}
Purpose: Computer-aided methods for mandibular reconstruction have improved both functional and morphological results in patients who underwent segmental mandibular resection. The purpose of this study is to evaluate the overlaying of virtual planning in terms of measures of the Computer Assisted Design/Computer Assisted Manufacturing CAD/CAM plate for mandibular reconstruction in patients who are ineligible for the insertion of reconstructing the titanium plate supported by fibular free flap, due to their poor health status, or in the presence of specific contraindications to autologous bone flap harvest. Materials and methods: The retrospective study performed analyzed the results of nine patients. The patients were treated at the Maxillofacial Surgery Unit of Policlinico S. Orsola of Bologna, Italy, and Policlinico San Marco, Catania, Italy, from April 2016 to June 2021. Superimposition between planning and post operative Computed Tomography CT scan was performed to assess the accuracy. Results: All reconstructive procedures were carried out successfully. No microsurgery-related complications occurred. In two cases, we had plate misplacement, and in one case, plate exposure that led to plate removal. The average accuracy of the series assessed after CT superimposition, as previously described, was $0.95 \mathrm{~mm}$. Conclusions: Considering that microvascular bone transfer is a high-risk procedure in BRONJ patients, we can conclude that the positioning of a customized bridging mandibular prosthesis (CBMP), whether or not it is associated with a microvascular soft tissue transfer, is a safe technique in terms of surgical outcome and feasibility.
\end{abstract}

Keywords: mandibular reconstruction; microsurgery; CAD/CAM; customized plate; PSI

\section{Introduction}

Nowadays, thanks to the technological improvements developed in the past few years in support of maxillo-facial surgery, it is possible to obtain better aesthetic and functional results in challenging reconstructions as well, such as mandibular defects, and restore the aesthetic profile and functions performed by the mandible after ablative surgery [1]. The current gold standard for the restoration of extensive mandibular defects after surgical ablation is the insertion of a reconstructive titanium plate supporting a microvascular fibular free flap [2,3].

However, when it comes down to patients affected by osteonecrosis of the jaw (ONJ) patients, mandibular reconstruction becomes a different, and more difficult, challenge. 
ONJ is a disease with a wide variety of symptoms, such as pain, bone exposure, swelling, infection, and pathological fractures in the final stages. During recent years, a number of ONJ diseases have been described $[4,5]$.

These patients are often not eligible for bony free flap reconstruction, because of systemic contraindications (low general performance status) or donor-site contraindications to the harvest of microvascular bone flaps (peripheral arterial occlusive disease or vascular anomalies), lack of good quality cervical vessels, lupus anticoagulants. Moreover, these patients are not eligible for implant rehabilitation, so they do not require bony reconstruction.

For this reason, alternative reconstructive boneless procedures have been developed lately. Among the procedures proposed, a customized reconstructive mandibular prosthesis, with or without a soft tissue free flap, has become widely accepted [6,7].

These procedures have been confirmed as viable in restoring the morphology and function of the mandibular region [8], also improving the quality of life of the patients [9].

To the best of our knowledge, there are no studies in the present literature assessing the quantitative accuracy of these CAD/CAM boneless reconstructions.

The aim of the present paper is indeed to assess the accuracy of this type of reconstructions using a CAD/CAM mandibular prosthesis.

\section{Materials and Methods}

The current study was based on a retrospective multi centric analysis of patients who underwent mandibular reconstruction, benefiting from the application of a plate produced with computer-aided manufactory (CAD-CAM) technology, and for whom a bone free flap was contraindicated.

The patients were treated at the Maxillofacial Surgery Unit of Policlinico S. Orsola of Bologna, Italy, and Policlinico San Marco, Catania, Italy, from April 2016 to June 2021. The study was approved by the S. Orsola Hospital Ethics Committee (approval no. 57/2011/O/Disp).

The inclusion criteria were:

- $\quad$ Age $\geq 18$ years;

- $\quad$ Osteonecrosis of the mandible induced by biphosphonates;

- Clinical and radiological signs of mandibular involvement under the course of the mandibular canal;

- $\quad$ Contraindications to the use of reconstruction with bone-free flap transfer;

- $\quad$ Need of primary or secondary reconstruction of mandibular continuity defects;

- $\quad$ Reconstruction with customized mandibular bridging plate with or without flee flap transfer.

The exclusion criteria were:

- Reconstruction with a standard osteosynthesis plate, with or without bone-free flaps; - Osteoradionecrosis of the jaw.

All surgical and reconstructive procedures were virtually planned and performed according to our CAD-CAM protocol.

All patients underwent a CT scan examination and the images obtained were saved in DICOM (Digital Imaging and Communication in Medicine) format. Using the Mimics (Materialize) software, the images were segmented, and the bone tissue was isolated from the soft tissues. Segmented images made it possible to reconstruct the 3D model of each patient's bone tissue (Figure 1).

The STL files of the models obtained in the previous phase were imported into the 3-Matic (Materialize) design software. In the design of the device, the morphology of the bone defect, after the virtual resection, was recreated thanks to the superimposition of the healthy area, mirrored with respect to the sagittal plane (Figure 2). 


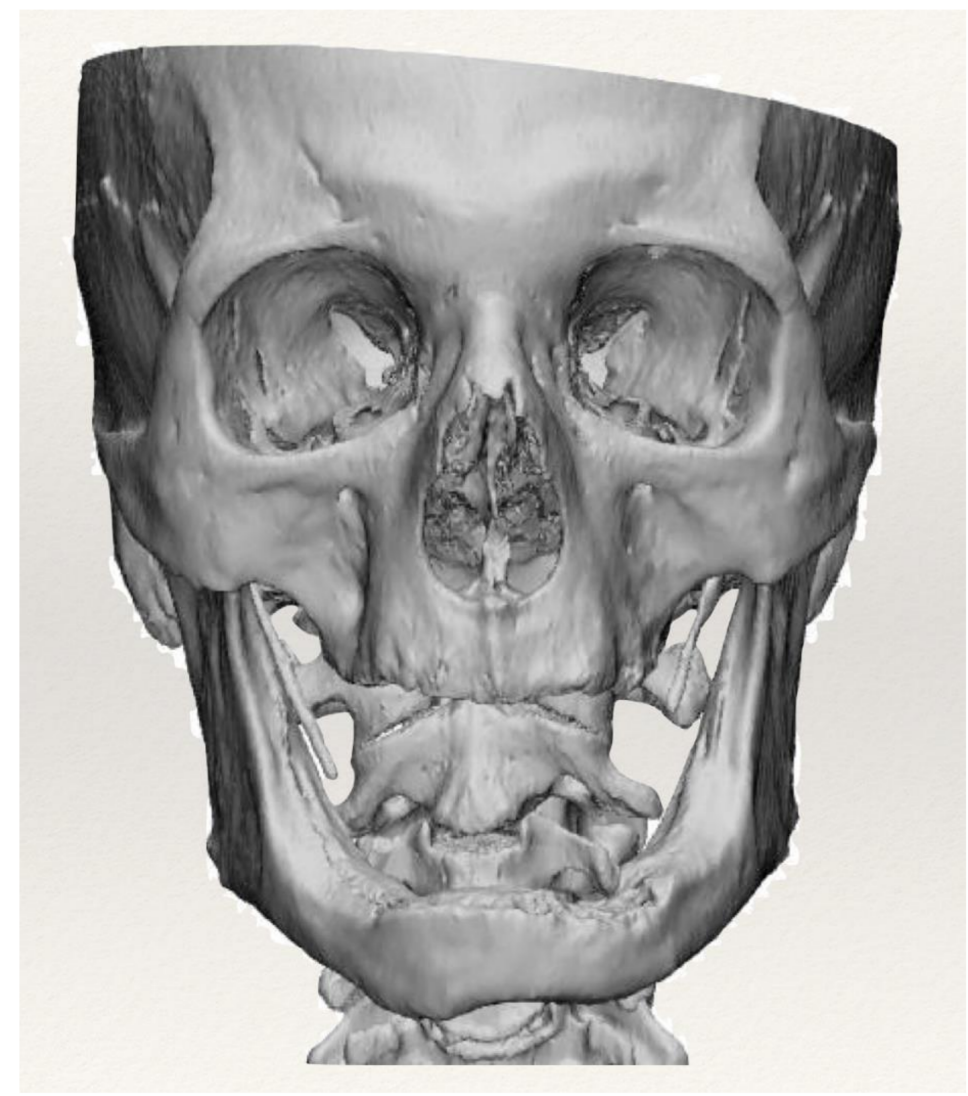

Figure 1. 3D model of the patient.

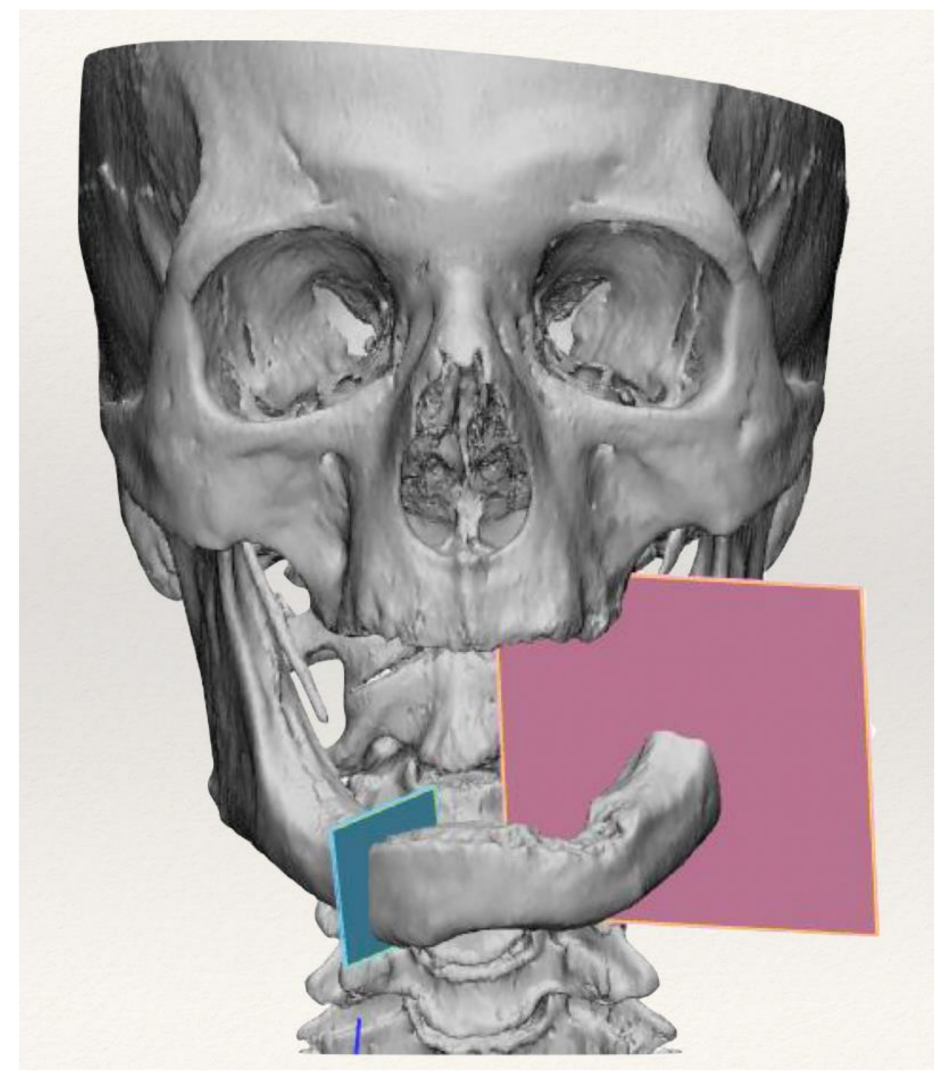

Figure 2. Virtual resection planning. 
The fixing system was created by designing anatomical plates which, by means of screws, ensure the stability of the implant. The preoperative planned resection was transferred into surgery using anatomical cutting guides. The unique positioning of the cutting guides ensures the execution of cuts with the orientation and position defined by the surgeon during the virtual simulation (Figure 3). Once the design phase was completed, the files were saved in STL format and processed to be produced using EBM (electron beam melting) additive technology from titanium alloy powder (Ti6Al4V-ELI).

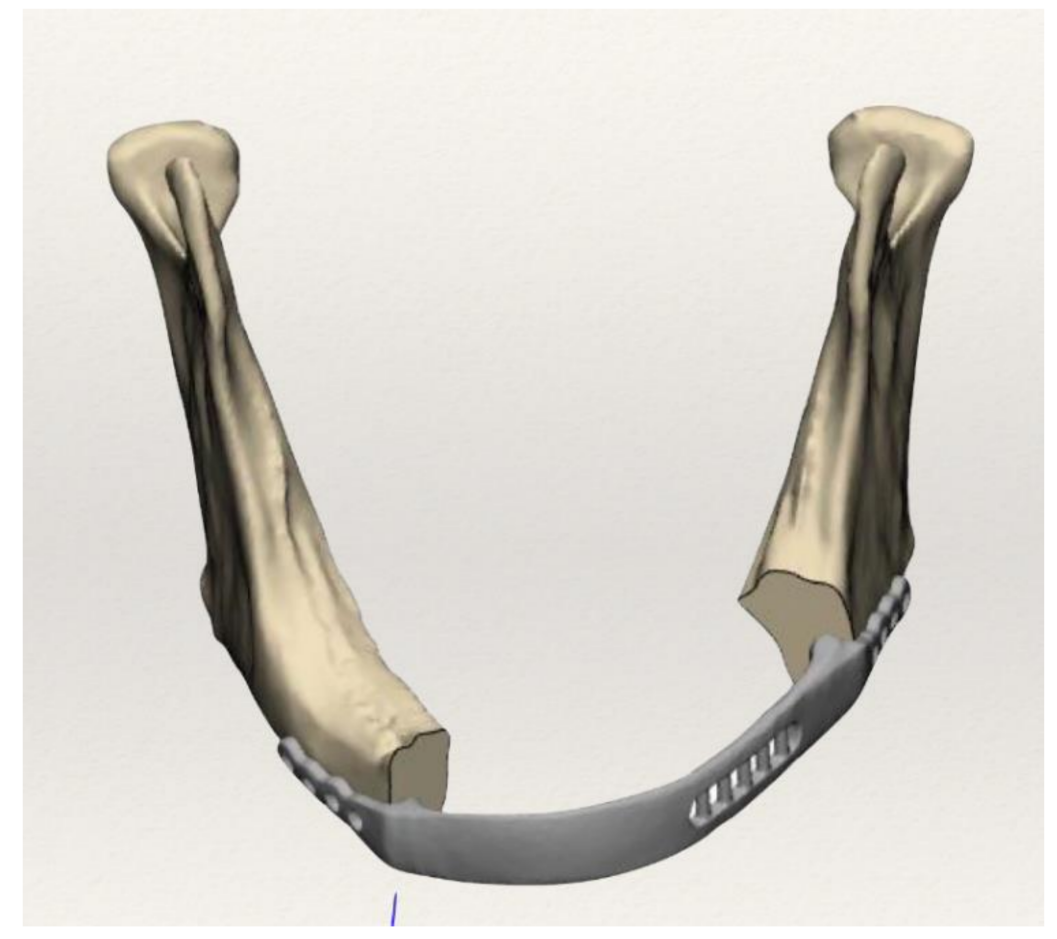

Figure 3. Virtual reconstruction. A grid is created in the body portion of the plate to anchor the oral pelvi muscles.

After surgery, all patients underwent a control CT examination, and with the same procedure, the 3D models of bone and implant were obtained. To assess the accuracy between preoperative planning and surgery, the models obtained were imported into the design environment. The "Create Part Comparison Analyzes" function of the Analyze window of the 3-Matic software allowed us to compare the 3D design models with the $3 \mathrm{D}$ reconstructions obtained from the postoperative CT images. The result of the analysis is a deviation in millimeters between the nodes of the triangles of the two models. This result can then be displayed on the 3D models thanks to a color scale or a histogram, which provides the number of points for each deviation value at a given slider thresholds value (Figure 4).

The surgical procedure was carried out, avoiding intra-oral access when possible. Through a cervicotomy access, the cutting guides were positioned and osteotomies performed as pre-operatively planned, using a piezoelectrical bone saw (Piezosurgery Medical, Mectron, Genoa, Italy). Then, the customized reconstructive plate was positioned to restore the mandibular anatomy, and fixed with 2.0 screws (Figure 5).

When, necessary, the end oral defect was reconstructed with a soft tissue free flap transfer. The oral pelvi muscles were anchored to a grid of the customized plate to allow a quicker swallowing functional recover.

Post-operatively, all patients underwent a CT scan two weeks after surgery, to assess the accuracy of the planned reconstruction as previously described. 


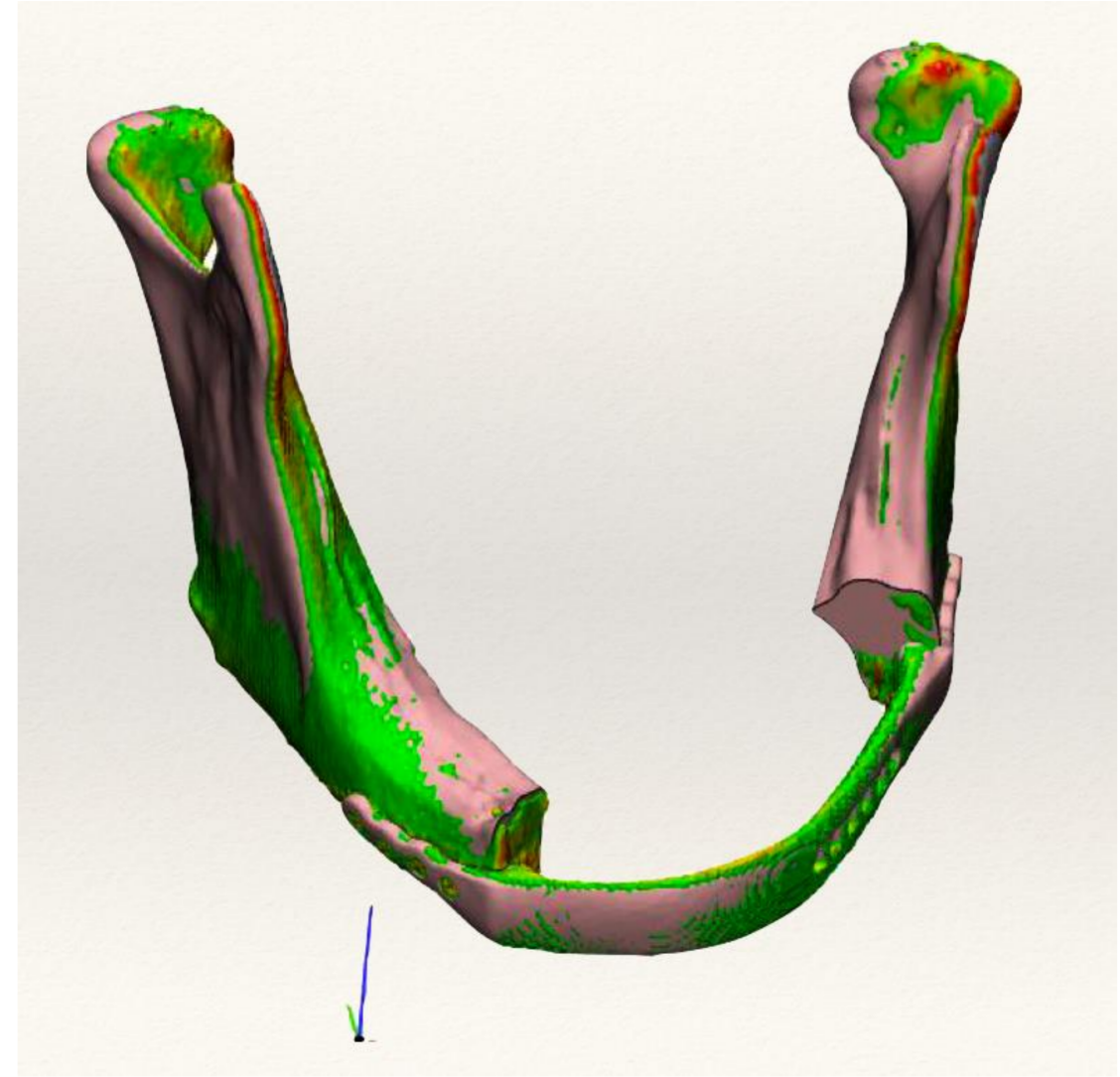

Figure 4. Superimposition of planning and post-operative CT scan obtaining a color map.

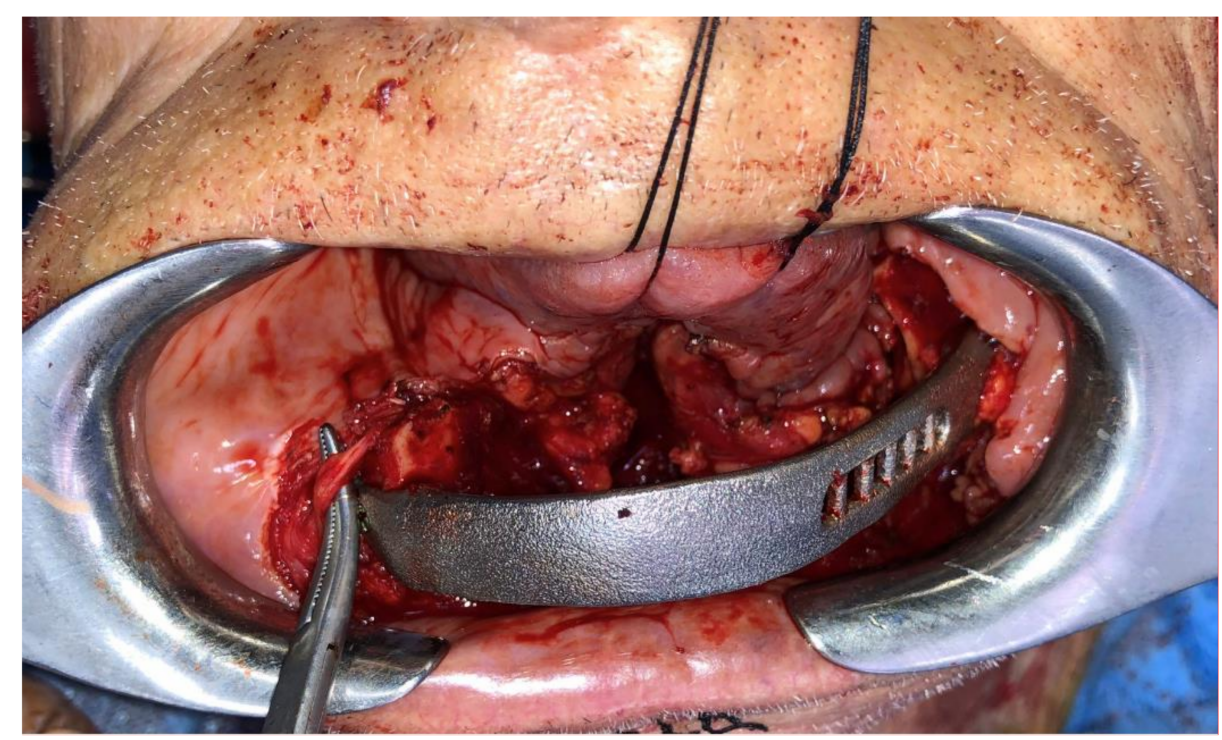

Figure 5. Fixation of the reconstructive plate.

\section{Results}

Between April 2016 and June 2021, we enrolled 10 patients according to the previously mentioned inclusion criteria; five treated at Policlinico S. Orsola, Bologna, Italy, and five treated at Policlinico San Marco, Catania, Italy.

Among all patients, four patients were affected by ONJ while 6 had BRONJ disease. In five cases, our procedure was secondary to complications from previous surgery (four 
standard plate rupture and one plate displacement). Five cases needed free tissue transfer (four anterolateral thigh flaps and one forearm flap). The average postoperative follow-up was 32.4 (range 6-64) months.

All reconstructive procedures were carried out successfully. No microsurgery-related complications occurred.

In two cases, we had plate misplacement, and in one case, plate exposure that led to plate removal.

The average accuracy of the series assessed after CT superimposition, as previously described, was $0.95 \mathrm{~mm}$ (range $0.57-1.45 \mathrm{~mm}$ ) (Figure 6). See Table 1 for summary results (Table 1).

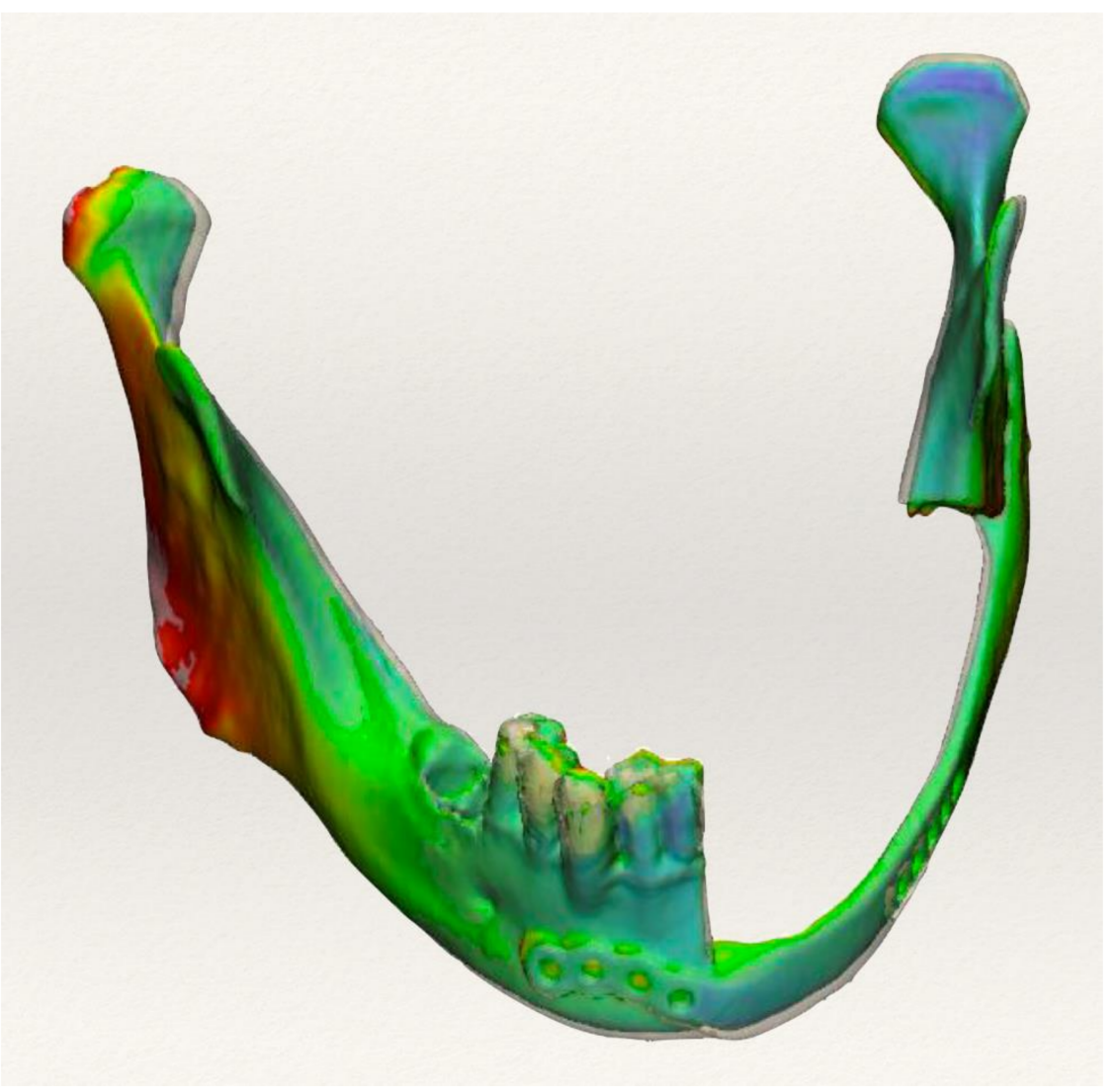

Figure 6. Superimposition and color map of accuracy. Green color represents best accuracy level. In green is displayed the best accuracy between planning and result, in red the worst accuracy obtained.

Table 1. Summary of the results of the study.

\begin{tabular}{cccc}
\hline ONJ (plate rupture) & CAD/CAM plate & $/$ & 1.03 \\
\hline BRONJ & CAD/CAM plate + ALT & $/$ & 0.87 \\
\hline BRONJ (plate rupture) & CAD/CAM plate & $/$ & 0.58 \\
\hline BRONJ (plate displacement) & CAD/CAM plate + ALT & Plate exposure & 1.45 \\
\hline ONJ & CAD/CAM plate + ALT & $/$ & 1.31 \\
\hline BRONJ (plate rupture) & CAD/CAM plate & Plate misplacement & 0.47 \\
\hline BRONJ (plate rupture) & CAD/CAM plate & $/$ & 1.07 \\
\hline ONJ & CAD/CAM plate + ALT & 1.22 \\
\hline BRONJ & CAD/CAM plate & 0.57
\end{tabular}




\section{Discussion}

The results of the case series reported in the present paper showed how it is possible to obtain accurate results in the mandibular reconstruction of ONJ-affected patients, using a bridging prosthesis as an alternative to bony reconstruction. It is well known that surgeons can benefit from CAD/CAM technology, as it improves precision, while also reducing morbidity and operational time [10]. This goal can be achieved by designing and manufacturing mandibular reconstructive plates and cutting guides that allow the surgeon to perform the virtually planned excision and reconstruction intraoperatively.

The data coming up from the accuracy study of these ten patients showed encouraging results in surgical terms, underlining the advantages that are derived from the use of just the plate, such as increased surgical preciseness from the use of the bone free flap technique, better procedural control, and reduced procedure time; in vivo site replication and the orientation of osteotomies thanks to surgical planning, the mandibular contour is reproduced exceedingly accurately without the necessity for manual remodeling of the reconstructive plate; the possibility to precisely adapt the virtual mandible both aesthetically and functionally considering the patient's occlusal features, even when the tumorous process has invested the mandible bilaterally, altering the regular mandibular morphology, thanks to the mirroring technique; the use of the first pre-operatory CT scan and/or the patient's native mandible printed in anticipation of an eventual accurate three-dimensional relocation of the interrelated mandibular segments, and of the same segments with the glenoid fossa.

In our study, the accuracy of reconstruction was assessed using the automated Hausdorff distance function and, as the difference between the postoperative condition and virtual planning, it was feasible to automatically determine the lowest, maximum, and average error for each reconstruction. As a result, the human error that may have influenced the manual measurements was eliminated. Although this is an instant and automatic surface assessment tool, the superimposition tool may underestimate errors when two complicated three-dimensional models are overlapped. When a reconstructive plate is used as a reference point, however, this method appears to be highly significant for evaluating surface superimposition, and it appears to be more efficient in representing the accuracy of mandibular reconstruction than a linear measurements assessment or point-based analysis. Finally, the data coming out of this short retrospective study suggest how CAD/CAM microvascular reconstructions can result in a high degree of reproducibility. It is, however, relevant to point out the fact that the maximum error between the original planning and the postoperative $\mathrm{CT}$ scans is located in the symphysis area, while the remaining mandibular parts are characterized by a high degree of accuracy in reproducibility. This demonstrates the effectiveness of the CAD/CAM method in reconstructing complicated anatomical locations, such as the condyle and ramus areas. However, even considering the margin of error of the inaccurate overlap in the parasymphisis area, the surgical outcomes are still significant both from an aesthetical and functional point of view, overcoming the general comorbidities contra-indications.

\section{Conclusions}

Considering the fact that microvascular bone transfer is a high-risk procedure in BRONJ patients, we can conclude that the positioning of a customized bridging mandibular prosthesis (CBMP), whether or not it is associated with a microvascular soft tissue transfer, is a safe technique in terms of surgical outcome and feasibility.

Author Contributions: Conceptualization, S.B. and S.C.; methodology, A.B.; software, R.M. and F.M.; validation, A.B., A.T. and S.C.; formal analysis, C.M.; investigation, F.R.; resources, S.C.; data curation, S.B.; writing—original draft preparation, S.B.; writing—review and editing, S.C.; visualization, A.B.; supervision, C.M. All authors have read and agreed to the published version of the manuscript.

Funding: This research received no external funding.

Institutional Review Board Statement: Not applicable. 
Informed Consent Statement: Informed consent was obtained from all subjects involved in the study.

Data Availability Statement: The data presented in this study are available in the present paper and in the archives of Policlinico San Marco di Catania.

Conflicts of Interest: The authors declare no conflict of interest.

\section{References}

1. Tarsitano, A.; Ciocca, L.; Scotti, R.; Marchetti, C. Morphological results of customized microvascular mandibular reconstruction: A comparative study. J. Cranio-Maxillofacial Surg. 2016, 44, 697-702. [CrossRef] [PubMed]

2. Brown, J.; Lowe, D.; Kanatas, A.; Schache, A. Mandibular reconstruction with vascularised bone flaps: A systematic review over 25 years. Br. J. Oral Maxillofac. Surg. 2017, 55, 113-126. [CrossRef] [PubMed]

3. Kumar, B.P.; Venkatesh, V.; Kumar, K.A.J.; Yadav, B.Y.; Mohan, S.R. Mandibular Reconstruction: Overview. J. Maxillofac. Oral Surg. 2015, 15, 425-441. [CrossRef]

4. Marx, R.E. A new concept in the treatment of osteoradionecrosis. J. Oral Maxillofac. Surg. 1983, 41, 351-357. [CrossRef]

5. Durie, B.G.; Katz, M.; Crowley, J. Osteonecrosis of the jaw and bisphosphonates. N. Engl. J. Med. 2005, 353, 99-102. [PubMed]

6. Bedogni, A.; Bettini, G.; Ferronato, G.; Fusetti, S.; Saia, G. Replacement of fractured reconstruction plate with customized mandible implant: A novel technique. Laryngoscope 2014, 124, 401-404. [CrossRef]

7. Tarsitano, A.; Battaglia, S.; Sandi, A.; Marchetti, C. Design of a customised bridging mandibular prosthesis for complex reconstruction: A pilot study. Acta Otorhinolaryngol. Ital. 2017, 37, 195-200. [CrossRef] [PubMed]

8. Ricotta, F.; Battaglia, S.; Bolognesi, F.; Ceccariglia, F.; Marchetti, C.; Tarsitano, A. Use of CAD-CAM Bridging Mandibular Prosthesis in Osteonecrosis of the Jaw: The Experience of Our School. J. Clin. Med. 2020, 9, 3516. [CrossRef] [PubMed]

9. Bedogni, A.; Bettini, G.; Bedogni, G.; Menapace, G.; Sandi, A.; Michelon, F.; Di Carlo, R.; Franco, P.; Saia, G. Safety of boneless reconstruction of the mandible with a CAD/CAM designed titanium device: The replica cohort study. Oral Oncol. 2021, 112, 105073. [CrossRef] [PubMed]

10. Tarsitano, A.; Battaglia, S.; Crimi, S.; Ciocca, L.; Scotti, R.; Marchetti, C. Is a computer-assisted design and computer-assisted manufacturing method for mandibular reconstruction economically viable? J. Cranio-Maxillofacial Surg. 2016, 44, 795-799. [CrossRef] [PubMed] 